BMJ Open Ophthalmology

\title{
Ophthalmic simulated surgical competency assessment rubric (Sim- OSSCAR) for trabeculectomy
}

\author{
William H Dean, ${ }^{01,2}$ John Buchan, ${ }^{1}$ Fisseha Admassu, ${ }^{3}$ Min J Kim, ${ }^{4}$ Karl C Golnik, ${ }^{5}$ \\ Andrew McNaught, ${ }^{6}$ Matthew Burton ${ }^{1,7}$
}

To cite: Dean WH, Buchan J, Admassu F, et al. Ophthalmic simulated surgical competency assessment rubric (SimOSSCAR) for trabeculectomy. BMJ Open Ophthalmology 2019;4:e000313. doi:10.1136/ bmjophth-2019-000313

Received 2 April 2019 Revised 31 July 2019 Accepted 3 August 2019
Check for updates

(C) Author(s) (or their employer(s)) 2019. Re-use permitted under CC BY. Published by BMJ.

${ }^{1}$ International Centre for Eye Health, London School of Hygiene and Tropical Medicine, London, UK

${ }^{2}$ Division of Ophthalmology, University of Cape Town, Cape Town, South Africa

${ }^{3}$ Ophthalmology, Gondar College of Medical Sciences, Gondar, Ethiopia

${ }^{4}$ Faculty of Infectious and Tropical Diseases, London School of Hygiene and Tropical Medicine, London, UK ${ }^{5}$ International Council of Ophthalmology, San Francisco, California, USA

${ }^{6}$ Ophthalmology, Gloucestershire Hospitals NHS Foundation Trust, Cheltenham, UK

${ }^{7}$ Moorfields Eye Hospital, London, United Kingdom

Correspondence to Dr William H Dean; whd1_uk@ hotmail.com

\section{ABSTRACT}

Background/aims To develop, test and determine whether a surgical-competency assessment tool for simulated glaucoma surgery is valid.

Methods The trabeculectomy ophthalmic simulated surgical competency assessment rubric (Sim-OSSCAR) was assessed for face and content validity with a large international group of expert eye surgeons. Cohorts of novice and competent surgeons were invited to perform anonymised simulation trabeculectomy surgery, which was marked using the Sim-OSSCAR in a masked fashion by a panel of four expert surgeons. Construct validity was assessed using a Wilcoxon rank-sum test. Krippendorff's alpha was calculated for interobserver reliability.

Results For the Sim-OSSCAR for trabeculectomy, 58 of 67 surgeons ( $86.6 \%$ ) either agreed or strongly agreed that the Sim-OSSCAR is an appropriate way to assess trainees' surgical skill. Face validity was rated as 4.04 (out of 5.00). Fifty-seven of 71 surgeons (80.3\%) either agreed or strongly agreed that the Sim-OSSCAR contents represented the surgical technique of surgical trabeculectomy. Content validity was rated as 4.00 . Wilcoxon rank-sum test showed that competent surgeons perform better than novices $(p=0.02)$. Interobserver reliability was rated $>0.60$ (Krippendorff's alpha) in 19 of 20 steps of the Sim-0SSCAR.

Conclusion The Sim-OSSCAR for trabeculectomy, a newly developed and validated assessment tool for simulation glaucoma surgery, has validity and reliability. It has the potential to play a useful role in ophthalmic surgical education.

\section{INTRODUCTION}

Glaucoma is the third most common cause of blindness globally after cataract and uncorrected refractive error. ${ }^{1}$ Surgical treatment for glaucoma is considered when medical and laser treatment options are exhausted, inappropriate, or unavailable. In many instances, surgical trabeculectomy is considered as a first-line treatment for moderate to advanced glaucoma. Early surgery can provide lower intraocular pressure (IOP) than medical therapy. ${ }^{23} \mathrm{~A}$ prospective multicentre randomised controlled trial is currently underway to compare the effectiveness of primary medical and primary surgical management for people presenting

\section{Key messages}

What is already known about this subject?

- A surgical competency assessment tool has already been developed and validated for live glaucoma surgery.

\section{What are the new findings?}

$>$ A new surgical competency assessment tool has been developed and validated for use in initial simulation-based surgical training in glaucoma surgery.

How might these results change the focus of research or clinical practice?

Ophthalmology training institutions might focus on the use of simulation-based acquisition of surgical competence before live surgical training.

with advanced glaucoma, the Treatment of Advanced Glaucoma Study. ${ }^{4}$

Surgical education for glaucoma is challenging. Opportunities for trainees are often sparse. In the USA, the mean number of trabeculectomies performed by trainees is four. ${ }^{5}$ Similarly, in sub-Saharan Africa the mean number performed by senior trainees was also four (article under review). This may be due to reluctance of surgeons to perform and patients to accept surgery, driven at least in part by the lack of expectation of improvement in vision and visual field loss. Vision never improves, and often is slightly worse following surgery: a recent meta-analysis showed that visual function (mean deviation and best-corrected visual acuity) drops after surgery, however, the gains from reduced rate of progression balance after 18 months, leaving patients better off. ${ }^{6}$ Moreover, the operated eye may be an only eye, often with good visual acuity. There is recent evidence that visual field loss can improve after surgery reduces the IOP. ${ }^{7}$

A structured curriculum, involving extensive simulation-based training, can assist in introducing trainees to glaucoma surgery. ${ }^{5}$ However, there is a paucity of data on the efficacy of simulation-based surgical education in 
glaucoma surgery techniques, including trabeculectomy. Therefore, to begin to address this gap, we designed a surgical competency assessment tool for simulated trabeculectomy surgery, based on the International Council of Ophthalmology (ICO) ophthalmology surgical competency assessment rubric (OSCAR) for trabeculectomy. ${ }^{8}$

Surgeons begin their training in a specific technique as 'novices', having incomplete knowledge and understanding, approaching a task relatively mechanistically. After time observing, learning and practicing under supervision a novice may progress to being an 'advanced beginner', demonstrating situational awareness and a working understanding of what is before them. They tend to see actions as a series of separated steps, and can complete some simpler surgical steps without supervision. A surgeon who is 'competent' in a technique has a good working and background understanding, and sees actions in relation to goals, at least partly in context. They may complete work independently to a standard that is acceptable, though it may lack refinement. They are capable of deliberate planning and can formulate surgical routines. ${ }^{9}$ Proficiency and full expertise are considered outside of the scope of this context of simulation-based surgical education in trabeculectomy. Even after an ophthalmology trainee has completed training, there is still a great amount of continued training and experience to be gained in order to become a glaucoma 'specialist' and attain a level to be considered and gain recognition as an 'expert'. ${ }^{10}$

It is towards the stage of 'competent' through structured ophthalmic surgical training that this development and use of the ophthalmic simulated surgical competency assessment rubric for trabeculectomy (Sim-OSSCAR) is designed to support. The Sim-OSSCAR is aimed at evaluating the progress made by a trainee towards a basic level of competence, in a simulation environment. Specifically, it addresses the binary question: has the trainee invested sufficient sustained deliberate practice on artificial materials for the trainer to decide it is reasonable to progress to supervised live surgical training?

In medical and surgical education, validity refers to the degree to which an instrument measures what it sets out to measure. Face validity describes whether the simulated tasks resemble those that are performed during a surgical procedure in a real-life situation. Content validity is whether the test resembles a specific skill, not other aspects such as anatomical knowledge. Intergrader reliability is the degree of agreement among different graders, and will provide a measure of consensus.

It is accepted that a unified approach of demonstrating evidence to either support or refute the overall validity of an instrument should be used. ${ }^{11}$ Studies of the assessment of surgical education, training and curricula should 
have discrete benchmarks as guides: described as face, content, construct, concurrent, discriminative and predictive validity. ${ }^{12}$ There is an even greater need for this in high-stakes assessments such as Board or Surgical College certification examinations. The ICO OSCAR for trabeculectomy has been validated for live surgical performance assessment. ${ }^{8}$ This current study is not aimed at validation of a curriculum nor a high-stakes live surgical assessment.

In this study, we aimed to modify the ICO OSCAR, using it as a starting point for developing a formative and summative assessment tool for simulated ophthalmic surgical training in trabeculectomy surgery.

\section{METHODS}

\section{Trabeculectomy Sim-OSSCAR content revision and development}

The ICO OSCAR for trabeculectomy was previously developed by experts at the ICO using a modified Dreyfus scale (novice, beginner, advanced beginner and competent) ${ }^{89}$ In this study, we have modified the original ICO OSCAR to develop an assessment and training tool for simulated ophthalmic surgical education in trabeculectomy surgery.

The ICO OSCAR was initially edited to remove content not appropriate for simulation-based surgical training. The OSCAR was further adapted to a modified three-stage Dreyfus scale (novice, advanced beginner, competent). The 'proficient' and 'expert' steps of the scale were excluded. The draft of the trabeculectomy Sim-OSSCAR was sent electronically to a panel of four international content experts for further amendments to the content and structure of the Sim-OSSCAR. These people were selected for their experience and expertise in performing and teaching trabeculectomy surgery. Responses were collated electronically and synthesised into a final version of the rubric, which was distributed for further review. Amendments suggested by only one of the four experts, and disagreements were discussed until a majority consensus was reached.

The Sim-OSSCAR was designed to be used in conjunction with artificial eyes specifically developed for trabeculectomy by Phillips Studios (Bristol, UK) ${ }^{13}$ which has been in used in training programmes for the past 6 years. It could be used in conjunction with surgical training using animal eyes.

\section{Face and content validity assessment}

The Sim-OSSCAR together with the artificial eye for glaucoma surgery, were presented to delegates at the International Glaucoma Society meeting in Germany. Delegates included expert glaucoma surgeons from around the world, and all were consultant or fellow

\begin{tabular}{|c|c|c|c|c|}
\hline 10 & $\begin{array}{l}\text { Formation of } \\
\text { sclerostomy with } \\
\text { punch/blade. }\end{array}$ & $\begin{array}{l}\text { Unable to insert Kelly's punch to perform } \\
\text { sclerostomy / to complete sclerostomy with } \\
\text { blade. No sclerostomy made. }\end{array}$ & $\begin{array}{l}\text { Able to use punch/blade to form } \\
\text { sclerostomy, though hesitant. Sclerostomy } \\
\text { too small/large. }\end{array}$ & $\begin{array}{l}\text { Able to use punch/blade to form a full } \\
\text { thickness sclerostomy. Correct size and } \\
\text { anterior position. }\end{array}$ \\
\hline 11 & Peripheral iridectomy & $\begin{array}{l}\text { Unable to retract iris and perform } \\
\text { iridectomy. }\end{array}$ & $\begin{array}{l}\text { Able to retract iris, but unable to complete } \\
\text { iridectomy, or iridectomy too anterior. }\end{array}$ & Able to retract iris, perform iridectomy. \\
\hline 12 & $\begin{array}{l}\text { Reformation of AC } \\
\text { using BSS via } \\
\text { paracentesis, } \\
\text { titration of IOP }\end{array}$ & $\begin{array}{l}\text { Failure to reform } \mathrm{AC} \text { with } \mathrm{BSS} \text {. } \\
\text { Failure to adjust tightness of releasable / } \\
\text { fixed sutures adequately. }\end{array}$ & $\begin{array}{l}\text { AC successfully reformed with BSS, but } \\
\text { failure to check IOP too high (via digital } \\
\text { IOP estimation), and need to release IOP } \\
\text { via paracentesis. }\end{array}$ & $\begin{array}{l}\text { AC efficiently reformed BSS, scleral flap } \\
\text { confirmed to be watertight efficiently, IOP } \\
\text { not excessive (efficient estimation of IOP } \\
\text { via digital pressure), but if so, IOP reduced } \\
\text { via efficient release of aqueous via } \\
\text { paracentesis / adjustment of sutures. }\end{array}$ \\
\hline 13 & Conjunctival suturing & $\begin{array}{l}\text { Unable to place and tie conjunctival } \\
\text { sutures, or places only one suture. }\end{array}$ & $\begin{array}{l}\text { Is able to eventually place and tie } \\
\text { conjunctival sutures, but inefficient / } \\
\text { multiple attempts. Places only two sutures }\end{array}$ & $\begin{array}{l}\text { Is able to efficiently place and tie } \\
\text { conjunctival sutures. Places three or more } \\
\text { sutures. }\end{array}$ \\
\hline 14 & $\begin{array}{l}\text { Conjunctival suture - } \\
\text { burying of knots }\end{array}$ & $\begin{array}{l}\text { No attempt made to bury conjunctival } \\
\text { suture knots. }\end{array}$ & $\begin{array}{l}\text { Attempts made to bury conjunctival knots, } \\
\text { but inefficient, suture snaps, or unable to } \\
\text { bury more than one knot. }\end{array}$ & $\begin{array}{l}\text { Fluent attempt made to bury conjunctival } \\
\text { knots along correct line of suture. Able to } \\
\text { rotate and bury two or more knots. }\end{array}$ \\
\hline \multicolumn{5}{|c|}{ GLOBAL INDICES } \\
\hline 15 & Tissue handling & $\begin{array}{l}\text { Tissue handling is often unsafe with } \\
\text { inadvertent damage, or excessively } \\
\text { aggressive or timid. }\end{array}$ & $\begin{array}{l}\text { Tissue handling is safe but sometimes } \\
\text { requires multiple attempts to achieve } \\
\text { desired manipulation of tissue. }\end{array}$ & $\begin{array}{l}\text { Tissue handling is efficient, fluid and } \\
\text { almost always achieves desired tissue } \\
\text { manipulation on first attempt. }\end{array}$ \\
\hline 16 & $\begin{array}{l}\text { Surgical Field } \\
\text { Positioned Centrally } \\
\text { Within Microscope } \\
\text { View }\end{array}$ & $\begin{array}{l}\text { Very limited or delayed repositioning. } \\
\text { Surgical operating field often at periphery } \\
\text { of microscope view. }\end{array}$ & $\begin{array}{l}\text { Surgical operating field occasionally at } \\
\text { periphery of microscope view. }\end{array}$ & $\begin{array}{l}\text { Surgical operating field occasionally at } \\
\text { periphery of microscope view. Adjusts } \\
\text { microscope as needed without delay. }\end{array}$ \\
\hline 17 & $\begin{array}{l}\text { Technique of holding } \\
\text { suture needle in } \\
\text { needle holder }\end{array}$ & $\begin{array}{l}\text { Loads needle in proper direction for fore- } \\
\text { hand pass but sometimes loads incorrectly } \\
\text { for backhand pass. Loads too close or too } \\
\text { far from the swaged end of needle. }\end{array}$ & $\begin{array}{l}\text { Loads needle properly for forehand and } \\
\text { backhand needle pass but is inefficient and } \\
\text { often requires multiple attempts. }\end{array}$ & $\begin{array}{l}\text { Loads needle properly and efficiently for } \\
\text { forehand and backhand needle passes. }\end{array}$ \\
\hline 18 & $\begin{array}{l}\text { Technique of surgical } \\
\text { knot tying }\end{array}$ & $\begin{array}{l}\text { Require multiple extra hand maneuvers to } \\
\text { make first throw lay flat, and/or loosens first } \\
\text { throw while attempting second throw. }\end{array}$ & $\begin{array}{l}\text { Is able to tie a flat surgeon's knot first throw } \\
\text { but second and third throws are inefficient. } \\
\text { Does not inadvertently loosen first throw. }\end{array}$ & $\begin{array}{l}\text { Is able to efficiently tie a flat, square } \\
\text { surgeon's knot. }\end{array}$ \\
\hline 19 & $\begin{array}{l}\text { Overall fluidity of } \\
\text { procedure }\end{array}$ & $\begin{array}{l}\text { Hesitant, frequent starts and stops. Not at } \\
\text { all fluid }\end{array}$ & $\begin{array}{l}\text { Occasional inefficient and/or unnecessary } \\
\text { movements or manipulations occur. }\end{array}$ & $\begin{array}{l}\text { Inefficient and/or unnecessary } \\
\text { manipulations are avoided. }\end{array}$ \\
\hline 20 & $\begin{array}{l}\text { Overall speed of the } \\
\text { procedure }\end{array}$ & $\begin{array}{l}\text { Case duration more than } 45 \text { minutes; or } \\
\text { case not completed. }\end{array}$ & Case duration between 30 and 45 minutes. & Case duration less than 30 minutes \\
\hline
\end{tabular}

Suggestions for development:

Figure 2 Ophthalmic simulated surgical competency assessment rubric: trabeculectomy (Sim-OSSCAR: Trab). IOP, intraocular pressure; BSS, balanced salt solution . 
ophthalmologists with a subspecialty interest in glaucoma. Questions were asked about the trabeculectomy Sim-OSSCAR regarding face and content validity. On a five-point Likert scale, surgeons were asked "Do you think the OSSCAR represents the surgical techniques and skills upon which trainees should be assessed?". Surgeons were also asked: "Do you think the Sim-OSSCAR (used with the artificial eye) is an appropriate way to assess trainees' surgical skill?". Responses on the five-point Likert scale were given a numerical value, entered onto a Microsoft Excel spreadsheet, prior to calculating the mean.

\section{Interobserver reliability assessment}

To assess interobserver Sim-OSSCAR grading reliability we recorded eight simulated trabeculectomy procedures, which were performed by eight separate trainee ophthalmologists. Four were novice trainee surgeons (assisted in less than five trabeculectomies) and four were experienced ophthalmologists (performed more than 100 trabeculectomies). The procedures were performed on the trabeculectomy-specific artificial eye. The simulated surgery was recorded using an Axiocam ErC5reV2 camera mounted to a Stemi 305 desktop microscope (Zeiss, Oberkochen, Germany). The videos were anonymised so that the people doing the scoring were masked to the level of the trainee. The recordings were independently graded using the trabeculectomy Sim-OSSCAR by four ophthalmologists who are highly experienced in trabeculectomy surgery. Expert assessors were masked to the training status of the trainee. Krippendorff's alpha was calculated for interobserver agreement correlation of the trabeculectomy Sim-OSSCAR ordinal marking scale for each of the 20 sections (figures 1 and 2). Low interrater reliability was considered for values of $\alpha_{\mathrm{k}}<0.60 .{ }^{14} 15$
Wilcoxon rank-sum test was performed using the rank sum of the mean scores for novice and competent surgeons. All analysis was performed using Stata V.15.1.

\section{RESULTS}

Trabeculectomy Sim-OSSCAR content revision and development

The changes arising from the editing of the ICO-OSCAR are shown in table 1 . The steps of draping, traction suture, tenons dissection, haemostasis, application of antimetabolite, knowledge of instruments and communication with team were removed. The first stage of 'globe stabilisation' included only a clear-corneal traction suture, and not a superior-rectus suture. The expert review group provided feedback on the content of the trabeculectomy Sim-OSSCAR.

\section{Face and content validity}

Seventy-one surgeons from 22 countries responded to the first question regarding the content of the Sim-OSSCAR, of these $57(80.3 \%)$ either agreed or strongly agreed that the Sim-OSSCAR contents represented the surgical technique of surgical trabeculectomy. The mean content validity was rated as 4.00 (out of 5.00).

Sixty-seven surgeons responded to the second question regarding the face validity of the assessment tool, of $58(86.6 \%)$ either agreed or strongly agreed that the Sim-OSSCAR is an appropriate way to assess trainees' surgical skill. The mean face validity was 4.04 .

\section{Interobserver reliability}

Interobserver reliability was assessed by four expert trabeculectomy surgeons. Eight separate masked video

\begin{tabular}{|c|c|c|c|}
\hline Stage of procedure & Action & New Sim-OSSCAR & Comment \\
\hline Draping & Deleted & & \\
\hline $\begin{array}{l}\text { Corneal or superior rectus traction } \\
\text { suture }\end{array}$ & Deleted and edited & Globe stabilisation & \\
\hline $\begin{array}{l}\text { Conjunctival incision and Tenon's } \\
\text { dissection }\end{array}$ & Deleted and edited & Conjunctival peritomy & Tenon's dissection deleted \\
\hline Maintaining haemostasis & Deleted & & \\
\hline Application of antimetabolite & Deleted & & \\
\hline \multicolumn{4}{|c|}{$\begin{array}{l}\text { Full thickness incision into anterior Added } \\
\text { chamber (AC) }\end{array}$} \\
\hline $\begin{array}{l}\text { Scleral flap suturing/AC } \\
\text { reformation }\end{array}$ & $\begin{array}{l}\text { Split into separate } \\
\text { sections, edited }\end{array}$ & Releasable, interrupted sutures & Further $\mathrm{AC}$ reformation \\
\hline Conjunctival closure & Edited & Conjunctival suturing, burying & \\
\hline Knowledge of instruments & Deleted & & \\
\hline \multicolumn{4}{|c|}{ Communication with surgical team Deleted } \\
\hline $\begin{array}{l}\text { Overall speed and fluidity of } \\
\text { procedure }\end{array}$ & Edited & $\begin{array}{l}\text { Fluidity separate } \\
\text { Times changes }\end{array}$ & \\
\hline
\end{tabular}

ICO, International Council of Ophthalmology; OSCAR, ophthalmology surgical competency assessment rubric; Sim-OSSCAR, ophthalmic simulated surgical competency assessment rubric. 
Table 2 Inter-rater Krippendorff's alpha correlation for 20 facets of the Sim-OSSCAR

\begin{tabular}{|llll}
\hline & Item & Krippendorff's alpha & Per cent agreement \\
\hline 1 & Globe stabilisation & 0.902 & 0.934 \\
\hline 3 & Conjunctival peritomy & 0.666 & 0.792 \\
\hline 4 & Scleral incision & 0.895 & 0.938 \\
\hline 5 & Corneal groove(s) & 0.782 & 0.875 \\
\hline 6 & Paracentesis & 0.880 & 0.934 \\
\hline 7 & Formation of scleral flap & 0.782 & 0.875 \\
\hline 8 & Releasable suture & 0.755 & 0.854 \\
\hline 9 & Fixed/releasable suture & 0.635 & 0.750 \\
\hline 10 & Corneal incision into AC & 0.665 & 0.771 \\
\hline 11 & Sclerostomy & 0.796 & 0.875 \\
\hline 12 & Peripheral iridectomy & 0.782 & 0.875 \\
\hline 13 & Reformation of AC & 1.000 & 1.000 \\
\hline 14 & Conjunctival suturing & 0.696 & 0.792 \\
\hline & Suture burying & 0.673 & 0.792 \\
\hline 15 & Global indices & & \\
\hline 16 & Tissue handling & 0.787 & 0.854 \\
\hline 17 & Surgical field positioned centrally within microscope view & 0.512 & 0.667 \\
\hline 18 & Needle holding & 0.665 & 0.771 \\
\hline 19 & Knot tying & 0.639 & 0.771 \\
\hline 20 & Overall fluidity of procedure & 0.743 & 0.854 \\
\hline OC, & Overall speed of procedure & 1.000 & 1.000 \\
\hline
\end{tabular}

AC, anterior chamber; Sim-OSSCAR, ophthalmic simulated surgical competency assessment rubric.

recordings of simulation trabeculectomy were sent to each expert surgeon for scoring using the Sim-OSSCAR. The mean score for 'novices' was 4.2 (SD 0.9) and mean for 'competent' trabeculectomy surgeons was 33.4 (SD 1.8 ), out of a maximum score of 40 .

To assess the interobserver agreement on the specific items in the Sim-OSSCAR, we calculated Krippendorff's alpha. A value of 0.60 was deemed acceptable for newly developed rubric. ${ }^{14}{ }^{15}$ Table 2 illustrates the results for all 20 items in the Sim-OSSCAR, of which 19 exhibited an inter-rater agreement coefficient of $\alpha_{k}>0.60$. Only the positioning of the microscope view had a $\alpha_{k}<0.60$.

\section{Construct validity}

Construct validity is an assessment of the 'sharpness' of a tool: can it discriminate between two distinct groups. For this study these groups are the novice and competent surgeons. Table 3 illustrates the total score for each separate grader for all eight videos.

Novice surgeons were graded with a mean score range of $0.50-13.5$ (out of 40), with SD varying between graders' scores of 0.58-1.5. Competent surgeons were graded with a mean score range of 29.75-37.25 (SD varying from 1.50 to 2.52). A Wilcoxon rank-sum test showed that competent surgeons perform better than novices $(\mathrm{p}=0.02)$.

\section{DISCUSSION}

Glaucoma remains a major cause of vision impairment and blindness globally. Four million people have moderate or severe vision impairment, and 2.9 million are blind from glaucoma. ${ }^{1}$ Despite this major burden of disease, trainee eye surgeons perform few glaucoma surgeries during training. There are many challenges in surgical education, with increasing demands for patient

\begin{tabular}{|c|c|c|c|c|c|c|}
\hline \multirow[b]{2}{*}{ Video } & \multicolumn{4}{|c|}{ Grader score: $\mathrm{n} / 40$} & \multirow[b]{2}{*}{ Mean } & \multirow[b]{2}{*}{ SD } \\
\hline & A & B & C & D & & \\
\hline 1 & 2 & 0 & 1 & 3 & 1.50 & 1.20 \\
\hline 2 & 0 & 1 & 0 & 1 & 0.50 & 0.58 \\
\hline 3 & 14 & 12 & 14 & 14 & 13.50 & 1.00 \\
\hline 4 & 0 & 2 & 0 & 3 & 1.25 & 1.50 \\
\hline 5 & 34 & 32 & 32 & 28 & 31.5 & 2.52 \\
\hline 6 & 38 & 36 & 36 & 39 & 37.25 & 1.50 \\
\hline 7 & 29 & 29 & 32 & 29 & 29.75 & 1.50 \\
\hline 8 & 37 & 35 & 36 & 33 & 35.25 & 1.71 \\
\hline
\end{tabular}

Videos 1-4 were performed by novice surgeons, 5-8 by competent surgeons. Scores were out of a possible total of 40 . Four expert surgeons (A, B, C and D) graded all eight videos independently. 
throughput, and reducing opportunities for trainees' hands-on experience. ${ }^{16}$ These challenges are global. If adequate experience cannot be gained through operating, effective adjuncts should be found.

There has been an increase in the use of simulators in ophthalmic surgical training in the past years. ${ }^{17-19}$ This offers an environment in which learners can train until they reach specified levels of competency. ${ }^{16}$ Through simulation-based surgical education, permission to fail can be built into the learning process without risking patient safety. This is especially important in intricate and challenging microsurgical procedures such as trabeculectomy. Furthermore, patients may present with advanced glaucoma, having already lost the vision in one eye. Many glaucoma surgeries are performed on a patient's only eye.

A trainee should proceed to supervised surgery training on patients in theatre only after having attained a level of competence in the simulated setting. Therefore, a structured training programme needs to include the formal assessment of the performance of simulated surgery, using a validated tool such as the trabeculectomy Sim-OSSCAR. The specific aim of this training and assessment rubric is to help train an eye surgeon who is a novice in trabeculectomy, to a competent level, such that they can commence supervised live surgical training.

The trabeculectomy Sim-OSSCAR has good interobserver reliability. The one step of the rubric to be rated less than 0.6 was 'surgical field positioned centrally within microscope view'. This is likely due to the limitation of the Zeiss Stemi305 microscope which has a higher zoom when recording, relative to the surgeon's binocular view. Therefore, the recorded image does not fully reflect the surgeon's experience.

There are limitations with the use of the Sim-OSSCAR. Its use should be flexible depending on the simulation environment. For artificial eyes, certain amendments or allowances could be made. These may include adding additional text:

- Toothed forceps for peripheral iridectomy (PI) (rather than micro-notched or suture tying forceps).

- Use of larger sutures (8-0) for scleral and conjunctival suturing, and allowances for slipping.

- Larger sclerostomy (than the $0.5 \mathrm{~mm}$ or $1 \mathrm{~mm}$ in live surgery).

- Flap should be measured from the limbus, and not the conjunctival insertion (which is usually $1-2 \mathrm{~mm}$ form the limbus due to a small band of glue which secures the silk mesh used to simulate conjunctiva). Furthermore, the conjunctival sutures would therefore traverse the middle of the scleral flap.

- Conjunctival suture 'burying' includes starting the suture from underneath the conjunctiva.

The Sim-OSSCAR should aid initial acquisition of competence for the novice glaucoma surgeon. The goal should be to use it as a formative assessment tool within a simulation-based surgical training programme for trabeculectomy, to take a novice surgeon to the stage of competent. It could then be used as a summative assessment tool to give the green light to proceed to supervised live surgical training. It would be up to individual ophthalmic surgeon trainers or training institutions to benchmark appropriately. A guide could be a mean total score of $80 \%$ over three simulated cases, and none of the 20 individual steps scoring a 'zero'.

We anticipate that this newly developed and validated competency assessment tool will help trainees and trainers in overcoming the challenges of training in glaucoma surgery. Further rigorous validation studies should be conducted for the educational curricula for glaucoma surgical education as a whole.

Contributors WHD, JB, KCG, AMN and MB designed the study. All authors contributed to the conducting of the study. WHD and MJK analysed the results. All authors contributed to the draft of manuscript and editing of the final paper.

Funding British Council for the Prevention of Blindness, Ulverscroft Foundation, CBM. MJB is supported by the Wellcome Trust (207472/Z/17/Z). JCB is supported by the Queen Elizabeth Diamond Jubilee Trust through the Commonwealth Eye Health Consortium.

\section{Competing interests None declared.}

Patient consent for publication This is an ophthalmic surgical competency assessment rubric validation paper, and as such no patients or public were involved in the design or conduct of this study.

Ethics approval The validation study was approved by the Medicine Education Ethics Committee (MEEC), Faculty Education Office (Medicine), Imperial College, London (MEEC1415-12); and London School of Hygiene \& Tropical Medicine ethics committee (11 795)

Provenance and peer review Not commissioned; externally peer reviewed.

Open access This is an open access article distributed in accordance with the Creative Commons Attribution 4.0 Unported (CC BY 4.0) license, which permits others to copy, redistribute, remix, transform and build upon this work for any purpose, provided the original work is properly cited, a link to the licence is given, and indication of whether changes were made. See: https://creativecommons.org/ licenses/by/4.0/.

\section{REFERENCES}

1. Flaxman SR, Bourne RRA, Resnikoff S, et al. Global causes of blindness and distance vision impairment 1990-2020: a systematic review and meta-analysis. Lancet Glob Health 2017;5:e1221-34.

2. Jay JL, Murray SB. Early trabeculectomy versus conventional management in primary open angle glaucoma. Br J Ophthalmol 1988;72:881-9.

3. Parrish RK, Feuer WJ, Schiffman JC, et al. Five-Year follow-up optic disc findings of the Collaborative initial glaucoma treatment study. Am J Ophthalmol 2009;147:717-24.

4. King AJ, Fernie G, Azuara-Blanco A, et al. Treatment of advanced glaucoma study: a multicentre randomised controlled trial comparing primary medical treatment with primary trabeculectomy for people with newly diagnosed advanced glaucoma-study protocol. $\mathrm{Br} J$ Ophthalmol 2018;102:922-8.

5. Gedde SJ, Vinod K. Resident surgical training in glaucoma. Curr Opin Ophthalmol 2016;27:151-7.

6. Junoy Montolio FG, Müskens RPHM, Jansonius NM. Influence of glaucoma surgery on visual function: a clinical cohort study and meta-analysis. Acta Ophthalmol 2019;97:193-9.

7. Musch DC, Gillespie BW, Palmberg PF, et al. Visual field improvement in the Collaborative initial glaucoma treatment study. Am J Ophthalmol 2014;158:96-104.

8. Green CM, Salim S, Edward DP, et al. The ophthalmology surgical competency assessment rubric for trabeculectomy. J Glaucoma 2017;26:805-9.

9. Dreyfus SE, Dreyfus HL. A Five-Stage model of the mental activities involved in directed skill acquisition, 1980.

10. Ericsson KA. Deliberate practice and the acquisition and maintenance of expert performance in medicine and related domains. Acad Med 2004;79(10 Suppl):S70-81. 
11. Sweet RM, Hananel D, Lawrenz F. A unified approach to validation, reliability, and education study design for surgical technical skills training. Arch Surg 2010;145:197-201.

12. Gallagher AG, Ritter EM, Satava RM. Fundamental principles of validation, and reliability: rigorous science for the assessment of surgical education and training. Surg Endosc 2003;17:1525-9.

13. Phillips C. Ophthalmic simulated surgery: Phillips eye Studio. Available: http://www.phillipsstudio.co.uk/ [Accessed 1 May 2015].

14. Arezes $P$, de Carvalho PVR. Ergonomics and human factors in safety management. CRC Press, 2016.

15. Krippendorff KH. Content Analysis - 3rd Edition : an Introduction to Its Methodology. Thousand Oaks: SAGE Publications, 2013.
16. Kneebone RL, Practice KRL. Practice, rehearsal, and performance: an approach for simulation-based surgical and procedure training. JAMA 2009;302:1336-8.

17. Thomsen ASS, Subhi Y, Kiilgaard JF, et al. Update on simulationbased surgical training and assessment in ophthalmology: a systematic review. Ophthalmology 2015;122:1111-30.

18. Ahmed Y, Scott IU, Greenberg PB. A survey of the role of virtual surgery simulators in ophthalmic graduate medical education. Graefes Arch Clin Exp Ophthalmol 2011;249:1263-5.

19. Oetting TA. Surgical competency in residents. Curr Opin Ophthalmol 2009;20:56-60. 